\title{
Pronounced Enhancement in Radiosensitization of Esophagus Cancer Cultivated in Docosahexaenoic Acid Via The PPAR-y Activation
}

\section{Ying Yang}

Medical Collage of Soochow University

\section{Ying Xu}

Medical Collage of Soochow University

Congzhao Zhao

Medical Collage of Soochow University

Lirong Zhang

Medical Collage of Soochow University

Aslibek Nuerbol

Gaochun Peoples' Hospital, Affiliated Hospital of Nanjing Drum Tower Hospital

\section{Lili Wang}

Second Hospital of Soochow University

Yang Jiao ( $\boldsymbol{\sigma}$ jiaoyang@suda.edu.cn )

Medical Collage of Soochow University

\section{Research Article}

Keywords: Docosahexaenoic acid, Esophageal cancer, Prognosis, Radiosensitivity

Posted Date: November 19th, 2021

DOI: https://doi.org/10.21203/rs.3.rs-1088207/v1

License: (c) (1) This work is licensed under a Creative Commons Attribution 4.0 International License. Read Full License 


\section{Abstract}

Background: Docosahexaenoic acid (DHA) has been reported to slow the tumor growth and improve prognosis, and been used to co-operate with many other chemotherapy medicines. Up to now, survey focuses on the interaction between DHA and radiation is relatively modest. Our study sought to evaluate the changes of radiosensitivity caused by DHA on esophageal cancer cells. Besides, potential mechanism and molecular are needed to be explored.

Results囚DHA enhanced proliferation inhibition of irradiation on esophageal cancer cells. DNA damage, especially double-strand break, induced by irradiation, was sharply increased by DHA addition. DHA accelerated G2/M phase blocking, cell apoptosis and lipid peroxidation after radiotherapy. The similar outcomings were observed in mouse xenograft tumor experiment that the malignancy substantially dismissed after the combined treatment of DHA and irradiation. Moreover, PPAR - $\gamma$ was verified to increase post-treatment. Suppression of PPAR $-y$ would attenuate the radio sensitization triggered by DHA incompletely.

Conclusion $\mathbb{D} \mathrm{DH}$ could improve radio sensitivity in esophageal squamous cancer cells in vivo and in vitro by activating PPAR $-\gamma$. Due to its explicitly usage and convenient, DHA and other PPAR - $y$ agonists would serve as an adjuvant therapy before radiotherapy if the clinical trials indicated positive from now on.

\section{Introduction}

Esophageal cancer has become the $7^{\text {th }}$ commonest malignant solid tumor worldwide, but it has such a poor prognosis that just ranks at $6^{\text {th }}$ from the bottom. ${ }^{1,2}$ Its common histopathologic type can be classified into two main kinds: squamous cell carcinoma(ESCC) and adenocarcinoma(EA). The former one occupies absolute majorities before 1970s, since then, however, the incidence of esophageal adenocarcinoma rises year by year. ${ }^{3,4}$ Especially, in UK and some other Western Europe countries, the morbidity of EAC has caught up with ESCC. ${ }^{5}$ These risk factors which take main responsible for ESCC are listed like Alcohol and Smoking, in the meantime, Alcohol and smoking are also related to the onset of EA..$^{6,7}$ In addition, Barrett's esophagus and obesity are the other two main causes of EA. ${ }^{8,9}$ Most ESCC's predilection site is the middle and upper esophagus, but the EA primarily occurs in the gastro junction. These two kinds of esophageal carcinomas share some clinical signs with each other, such as: dysphagia, weight loss and thoracalgia.

Due to the insidious onset of esophagus tumor, the diagnosis is often delayed to middle and late period so that miss the optimal opportunity of treatment. Surgery is the traditional choose for therapy for it can put more concentration on the solid tumor than other means. However, the 5 -year overall survival is poor since the tumor cells would locally recur and distant metastasize after the primary surgical resection of cancer leisions. ${ }^{10,11}$ Historically, radiotherapy (RT) has been exploited as a valid treatment, particularly for those who is not applicable for surgery operations. Lately, there's some studies demonstrate that partial RT could control the primary tumor which combined with some other symptoms so as to prolong survival 
time somehow. ${ }^{12,13}$ However, it bothers clinicians that radiotherapy may produce some side effect in addition and its efficacy upon advanced esophagus tumors remains to be improved. ${ }^{14}$ Thus, RT could be an effective method to be took advantage of together with other auxiliary measures.

Docosahexaenoic acid (DHA) is a long-chain n-3 polyunsaturated acid (n-3 PUFAs) and enriched in marine food product such as kelp and abyssal fishes, together with linoleic acid family (n-6 PUFAs) constitute the unsaturated fatty acids. It has been confirmed definitely n-3 PUFAs have anticancer activity in many systems. ${ }^{15-17} n-3$ PUFAs can partly prevent tumorigenesis ${ }^{16}$, inhibit cancer cell proliferation ${ }^{18}$ and refrain tumor growth. ${ }^{19,20}$ After almost a decade of prospective cohort investigation carried out by USA National Cancer Institute, they found that marine food intake was associated with a $20-27 \%$ lower risk of EA and head-neck tumors (HNC), but not ESCC. ${ }^{21}$

In this study, we investigate whether DHA could cooperate with radiation, which is one of the mainly therapeutic methods, to improve the esophagus squamous cells death or inhibit its proliferation. Of note, our results indicated that DHA further promoted radiation-induced cell viability inhibition and cell death via prolonging $\mathrm{G} 2 / \mathrm{M}$ arrest and apoptosis somehow in vivo and in vitro. Consider together, our study provides us a clinical evidence manifesting that radiotherapy and DHA used in conjunction could be a novel remedy for ECCs treatment. Furthermore, PPA R-y ( peroxisome proliferators-activated receptors), a ligand -activated transcription factor which regulates lipid metabolism, ${ }^{22}$ was proposed that could take participant in this mechanism and its suppression will inhibit the radio sensitization of DHA in turn.

\section{Results}

DHA reduces cell viability after irradiation in esophagus cancer cells

First, to determine the concentration of PUFAs we used, the CCK-8 assay was performed to investigate the toxicity of PUFAs upon esophagus cells. TE-1 and TE-10 cells were cultured with different concentrations(0-200uM) of DHA and linoleic acid(LNA) for $24 \mathrm{~h}$. The esophagus cells viability was inhibited by DHA and LNA with a dose-dependent relationship according to the results somehow. (Figure $1 A-B)$ When the TE- 1 and TE-10 cells inhibiting rate reached $20 \%$, the concentrations of DHA are $100 \mathrm{uM}$ and $150 \mathrm{uM}$, the same concentrations of LNA were picked as the matched group.

To clarify whether DHA has a combination effect with IR, colony formation assay was carried out respectively on TE-1 and TE-10 cells with the indicated dose of DHA and LNA. With the dose of radiation given increased(0-4Gy) after incubation with DHA and LNA, the ability to form cells colony goes down. (Figure 1C) In the meantime, the sensitizer enhancement ratio was 1.520(P凶0.01) for TE-1 relative to untreated cells, the similar outcoming was obtained from TE-10 with the ratio of 1.225, which implicated the possible reciprocity between DHA and radiotherapy. (Figure 1D)

DHA and IR combination enhances DNA damage in esophagus cancer cells 
Not only the destruction of DNA repair could result in the cell proliferation declines, but also the aggravated DNA damage. We used the immunofluorescence staining to detect $\mathrm{\gamma}-\mathrm{H}_{2} \mathrm{AX}$ quantity, one of the most commonly used markers of DNA damage, as well as the Single cell gel electrophoresis( SCGE).TE-1 and TE-10 cells were well treated with DHA and LNA, then, the cells were immobilized and stained at different time point. All the groups generate biggest DNA fragmentation index at 0.5 h after the irradiation, the DHA and radiation combination reached higher fragment index than the others in both these two kinds of esophagus cancer cells. (Figure 2A-B) This observation was also confirmed in cells which were nuclear stained of DAPI, placed in an electric field for $45 \mathrm{~min}$, to form DNA electrophoresis. DHA and radiation combination group owns the highest Tail DNA\%, Tail moment and Olive moment. The DNA damage comes to the peak at $0.5 \mathrm{~h}$ after radiation can be concluded from these figures. (Figure 2CD)

\section{DHA increases G2/M-phase arrest and apoptosis caused by IR}

In order to investigate whether DHA proliferation suppression has something to do with cell cycles progression, 2Gy X-ray were implemented to TE-1 and TE-10 cells after pretreated with DHA and LNA for 24h. Radiation alone has a G2/M arrest effect upon esophagus cancer cells, while the combination of radiation and DHA brings a longer block to G2/M compared with the precious group.(Figure 3A-B)The same does not happen in the group of LNA and radiation united treatment.

Flow cytometry was used to measure the apoptosis taken place $24 \mathrm{~h}$ after TE- 1 and TE-10 get irradiation of $4 \mathrm{~Gy}, \mathrm{DHA}$ and radiation combination brings higher amount of apoptosis occurred in TE- 1 and TE-10 than sole DHA group and sole radiation group. (Figure 3C) Same as before, this circumstance does not repeat in LNA and radiation combination group. Moreover, the apoptosis population shows a downward trend. (Figure 3D)

We took advantage of BODIPY 510/590 dyestuff to explore the lipid peroxidation occurred after the irradiation. As shown in the result, Radiation induced lipid peroxidation in TE- 1 and TE-10 cells immediately, besides this, both DHA and LNA addition intensified the lipid peroxidation and the former combination was higher than the later one. (Figure 3E-F)

DHA and radiation combination augments the expression of PPAR $-y$

PPAR - $y$ is one of the nuclear ligands which can be activated by fatty acid. To investigate that whether the improvement of radiosensitivity in TE- 1 and TE-10 induced by DHA relates to PPAR - $\gamma$, western blot was used to detect the protein expression. As shown in the result, DHA alone did not apparently affect the expression, but the combination of DHA and IR did. The expression of PPAR - $\gamma$ was enhanced after irradiation, indicating an activation of PPAR-ץ. (Figure4A-B)

PPAR -y inhibition attenuates DHA -mediated radio sensitization 
To explore whether the radio sensitizing effect of DHA owes to PPAR - $y$, TE- 1 and TE-10 cells are respectively incubated with Mifobate and GW9662, two kinds of PPAR - $y$ antagonists, for 24h before applied with DHA and radiation. Firstly, we used CCK-8 assay to establish the working concentration of Mifobate and GW9662.(Figure4C-D) Then immunoblotting was carried out to guarantee that PPAR $-\gamma$ was suppressed, which was in line with our anticipation, the expression of PPAR $-\gamma$ accumulated in cell nucleus was markedly cut down.(Figure4G-H) The immunofluorescence assay was then repeated to observe the $\mathrm{Y}-\mathrm{H} 2 \mathrm{AX}$, a marked indicator of DNA damage, at $0.5 \mathrm{~h}$ after the treatment of 4Gy irradiation. The result shows that the fluorescent counting per cell increased at $0.5 \mathrm{~h}$ after the irradiation, which is consistent with our previous outcomes. Moreover, mifobate and Gw9662 twisted the tendency as mentioned earlier and Gw9662 played a stronger role. (Figure4E-F) Cell clones were generated after treatment of mifobate and Gw9662 for 24h prior to the interaction of DHA and radiation. The clone formation sharply decreased in the DHA and IR combination group, which is the same as what mentioned before. The effect of cell-killing was reversed in Mifobate and Gw9662 combined application groups, and the Gw9662 shows a more obvious resistant to the radiotherapy that contribute to the esophagus cancer cells proliferation. (Figure4I-J) Taken together, these results indicate that inhibition of PPAR $-y$ could relieve the DNA damage caused by DHA and IR co-treatment and relive the radio sensitization induced by DHA.

\section{DHA and radiation combination promotes cell death in vitro}

TE-1 cells $\left(1 \times 10^{7}\right.$ in $\left.100 \mu \mathrm{L}\right)$ were subcutaneously injected into five-week-old female nude mice and then be treated with $\mathrm{ddH}_{2} \mathrm{O}, \mathrm{DHA}$ and LNA by intragastric administration.(dosage regimen described in details later) The tumors were exposed to a 4Gy X-ray targeted radiotherapy after the treatment course. The DHA and radiation combination significantly slows down the tumor growth relative to the other groups. (Figure 5B) We performed an HE staining to compare the malignant degree among the control, DHA and LNA treatment groups. Consistent with the in vitro results, the DHA and radiation combination shows the lowest malignancy in comparison with other groups, which a few cancer cells can be found in the view of microscope. (Figure5C)

\section{Discussion}

Radiotherapy is a conventional clinical treatment for esophagus cancer. however, this treatment can cause some side-effect such as esophagitis and digestive symptoms. ${ }^{23}$ Besides, due to the esophagus cancer cells are not very sensitive to radiation, the curative effect of radiotherapy remains enhanced. Therefore, combination with other agents which could solve these problems were sought-after. N-3 PUFAs, especially DHA, have been proved to benefit to human body in many aspects. ${ }^{24,25}$ It is a dietary component and the amount of guidance is very clear. At the same time, DHA is well-focused recent years owing to its anti-tumor potential. ${ }^{15,16}$ What's more, several studies have proved that DHA can synergize with other drugs like Oxaliplatin, Adriamycin and Cisplatin nowadays, ${ }^{26-28}$ but there is still little research 
about the combination use of DHA and radiation. The current study investigated whether DHA have a reciprocity with radiation upon ECGG treatment.

Our results showed that DHA with radiation combination induced a decrease in cell viability in the ECGG cell lines. Interestingly, the other kind of PUFAs, LNA alone or with combination with radiation, did not trigger the same phenomenon. Considering that DHA could relieve inflammation and other side-effects caused by chemotherapy in esophago- gastric adenocarcinomas, it makes sense that DHA would enhance the efficacy of radiotherapy upon ECGG. ${ }^{29}$

DNA breakage is the main damage caused by radiation in cells, and the DNA double-strand breaks ( DSBs ) is the most lethal damage form. ${ }^{30}$ Here we used the $\mathrm{y}-\mathrm{H}_{2} \mathrm{AX}$ to mark the DSBs, which is the $\mathrm{Y}^{-}$ phosphorylation product of $\mathrm{H}_{2} \mathrm{AX}$ after irradiated and will be located to the break point. The population is positively associated with the number of DSBs. DHA increased the DSB induced by radiation while LNA did not. Contemporaneous with this result, comet trailing length of DHA and radiation combination group which was observed under the fluorescence microscope was the longest. Of note, both $\mathrm{Y}-\mathrm{H}_{2} \mathrm{AX}$ staining and comet assay indicated that DSB took shape quickly after the exposure and dismissed at $24 \mathrm{~h}$ by and large, which means DSB get repaired.

Once the destruction are repaired unsuccessfully, cells are subjected to begin programmed death, or apoptosis. ${ }^{31,32}$ Our data illustrated that DHA and radiation combination induced more apoptotic tumor cells via blocking the $\mathrm{G} 2 / \mathrm{M}$ phase, which is consistent with our previous study that DHA could arouse cell apoptosis in breast cancer cells and the human B-cell lymphoma lines. ${ }^{33}$ What may be responsible for the enhancement in apoptosis is the lipid peroxidation, as it was measured in this study, the DHA and radiation combination has sharply increased the level of lipid peroxidation compared with the control group. The LNA and radiation combination improved the lipid peroxidation level at the meantime, but not so efficient as DHA and radiation combination and didn't affect the cell viability according to our outcomings. Which could explain these differences between DHA and LNA is that DHA(n-3 PUFA) is more lipophilic than LNA(n-6 PUFA), represents faster incorporation into cell membrane and quicker oxidation. ${ }^{34}$ It is worth mentioning that compared with other n-3 PUFAs, DHA shows the strongest capability of tumor inhibition in breast cancer cell lines, seems to be proportional to their double bonds number. ${ }^{35}$

Thus far, DHA was reported to co-work with many chemical medicines to boost their antitumor activity as we mentioned before. ${ }^{36}$ Furthermore, DHA seems has no impact on the chemotherapy sensitization of normal tissues or non-tumor tissues. Consistently, our experiments indicate DHA could improve radiosensitivity of esophagus cancer cells. Notwithstanding numerous studies pointed out that cancer cells have many molecular targets, the studies upon DHA and PPAR - $y$ did not cohere with each other. ${ }^{37} 38$ The latest study upon a clinical trial conducted in Iran 2014 indicated that 8-weeks supplement of DHA will improve the PPAR - $y$ activity. Similarly, our results reveal that DHA remarkably aggravated PPAR $-\gamma$ expression of irradiated esophagus cancer cells. 
PPAR - $y$ is a widely distributed transcription factor. Several in vivo and in vitro studies have proved that PPAR $-\gamma$ is linked with a reduction of $\beta$-catenin levels, which plays a significant role in the tumorigenesis. ${ }^{39,40}$ Thus, PPAR $-\gamma$ seems to should be divided into the tumor suppressor genes family. DHA is a natural PPAR $-\gamma$ activator, so it is accessible to understand the reason why PPAR $-\gamma$ activated after the DHA supplementation esophagus cancer cell irradiated. Interestingly, the radiation sensitization of DHA was attenuated by PPAR-y inhibitor partly, which means PPAR- $\gamma$ may involve in the mechanism. More researches are necessary to figure out the specific pathway. Besides this, we are very curious about the variation of autophagy and immunogenic death caused by the combination of DHA and radiation.

PPAR - $y$ activators have been used as a monotherapy in several advanced carcinomas with no notable improvement been observed. ${ }^{41}$ Nonetheless, multimodality combination therapy is a tendency for nowadays co-therapy. We are next going to design experiment about the clinical trial of DHA and radiation combination therapy for the patients who with esophageal squamous cancers.

\section{Conclusions}

In conclusion, the present study focused on a potential combination therapy of irradiation and DHA. Moreover, a novel critical factor, PPAR - $\gamma$, was put forward to take participant into this synergism. As DHA is a convenient and safe health care product, a new adjuvant treatment is on the agenda as long as our following clinical trials suggest positively.

\section{Materials And Methods}

\section{Reagents and materials}

DHA, LNA were purchased from Sigma-aldrich(St Louis, MO, USA). CCK-8 kit was brought from Beyotime (Shanghai, China). The stock solution of DHA and LNA were prepared in corresponding solvent as described previously and all of these chemical ingredients are dissolved in the complete growth medium for working solutions. PPAR- $a, \beta$ and $\delta$ were ordered from Sigma-aldrich (St Louis, MO, USA).

\section{Cell line}

The human esophagus cancer cell line TE-1 and TE-10 were given from Professor Zhang in Suchow University as a gift. They were cultivated in RPMI Medium mixed with 10\% heat-inactivated foetal bovine serum and $2 \%$ Penicilin-streptomycin.

\section{Cell treatment with DHA/LNA together with irradiation}

Both TE-1 and TE-10 were treated with DHA/LNA for 24h before a single RT of 4Gy and the working concentrations were $150 \mathrm{Mmol}$ and $100 \mathrm{Mmol}$.

\section{Cell viability assay}


Cell viability was assessed using the CCK-8(cell counting kit 8) assay. Cells were plated into 96-well plates and the concentration was 6000/well. After 24h, cells were treated by DHA/LNA at $0,25,50,100$ and $200 \mathrm{uM}$ for another $24 \mathrm{~h}$. CCK- 8 was added into these plates and the cell viability was measured by Flow Cytometer since a 30 minutes incubation.

\section{Cell cloning assay}

The cloning experiment was carried out in the light of reported earlier. () Simply put, TE-1 and TE-10 cells were pretreated with DHA dissolved in medium for 24h, after that, $0,0.5,1,2,4 \mathrm{~Gy}$ single dose of X-ray were given to them.

\section{Immunofluorescence assay}

Immunofluorescence staining technique was used to detect the destruction of DNA and lipid peroxidation took place right after the irradiation. The fluorescent dyes used were as follows: DAPI, FITC, BODIPY.

\section{Western blot assay}

Western blot was performed as standard schedule. The antibodies which used in the experiment were PPAR- $\alpha$, PPAR- $\beta$ and PPAR- $\theta$. All the results were repeated three times. The relative grayscales of protein expression were normalized against the quantity of an internal control gene GAPDH (Santa Cruz Inc. California, USA).

\section{Human esophagus cancer xenograft mice experiment}

Five-week-old Balb/C nude mice(male) were purchased from Si-Laike Experimental animal Company(Shanghai, China) and used in this vivo experiment. All animal experiments complied with the ARRIVE guidelines and were carried out in accordance with the National Institutes of Health guide for the care and use of Laboratory animals (NIH Publications No. 8023, revised 1978). Every mouse were inoculated with TE-1 cells $\left(10 \times 10^{5}\right)$ subcutaneously and were randomly divided into six groups $(n=5)$. They received one of the following treatments while the tumors volume we manufactured before reached at $100 \mathrm{~mm}^{3}:$ (1)intragastric administration of $\mathrm{DHA}(500 \mathrm{mg} / \mathrm{Kg} \cdot \mathrm{d})$; (2)intragastric administration of $\mathrm{LNA}(500 \mathrm{mg} / \mathrm{Kg} \cdot \mathrm{d})$; (3) intragastric administration of $\mathrm{ddH}_{2} \mathrm{O}$; (4) the combination of DHA and irradiation; (5) the combination of LNA and irradiation; (6) the combination of $\mathrm{ddH}_{2} \mathrm{O}$ and irradiation. Tumors volume and mice weight were measured every 2 days right after treatment given and the Tumors got a single dose of 4Gy X-rays on day8.

\section{Hematoxylin and eosin (HE) staining}

The xenografts were fixed into $10 \%$ neutral-buffered formalin and paraffin embedded. The tumor blocks were cut apart into $4 \mu \mathrm{m}$ sections, then stained by HE dyestuff kit. (ZSGB-Bio, Beijing, China) The pathological sections were observed under Leica microscope. 


\section{Statistical analysis}

All of the values we mentioned before were expressed as mean \pm s.e.m. of three independent experiments. We used Graphpad Prism 8(Graphpad software, San Diego, CA, USA) to calculate the unpaired two-tailed Student's t-test and one-way analysis of variance. Survival curves were assessed on the basis of KaplanMeier method and compared using log-rank test. The difference among groups were regarded meaningful when the P-value was $<0.05$.

\section{Abbreviations}

DHA: docosahexaenoic acid, LNA: linoleic acid , ESCC: squamous cell carcinoma, EA: adenocarcinoma, RT: radiotherapy; PUFAs:polyunsaturated acid.

\section{Declarations}

\section{Ethics approval and consent to participate}

Not applicable.

\section{Consent for publication}

Not applicable.

\section{Availability of data and materials}

The datasets used and/or analysed during the current study are available from the corresponding author on reasonable request.

\section{Competing interests}

The authors declare that they have no competing interests.

\section{Funding}

Not applicable.

\section{Authors' contributions}

YY performed all the cell assays and the relative analysis, and also the major author of this manuscript; YX was responsible for the interpretation of the data; CCZ and LRZ took participant in the animal experiment; NA was responsible for data investigation; LLW helped designed the experiment methodology and the project administration; YJ designed these experiment schemes and revised this manuscript. All authors read and approved the final manuscript.

\section{Acknowledgments}


Not applicable.

\section{References}

1. Bollschweiler E, Plum P, Monig SP, Holscher AH. Current and future treatment options for esophageal cancer in the elderly. Expert Opin Pharmacother. 2017;18(10):1001-1010.

2. Kang M, Li Y, Zhu S, Zhang S, Guo S, Li P. MicroRNA-193b acts as a tumor suppressor gene in human esophageal squamous cell carcinoma via target regulation of KRAS. Oncol Lett. 2019;17(4):3965-3973.

3. Zhang M, Wu AJ. Radiation techniques for esophageal cancer. Chin Clin Oncol. 2017;6(5):45.

4. Fawaz ZS, Kazandjian S, Tsui JM, et al. What Is the Optimal Radiation Technique for Esophageal Cancer? A Dosimetric Comparison of Four Techniques. Cureus. 2018;10(7):e2985.

5. Bray F, Ferlay J, Soerjomataram I, Siegel RL, Torre LA, Jemal A. Global cancer statistics 2018: GLOBOCAN estimates of incidence and mortality worldwide for 36 cancers in 185 countries. CA Cancer J Clin. 2018;68(6):394-424.

6. Cook MB, Kamangar F, Whiteman DC, et al. Cigarette smoking and adenocarcinomas of the esophagus and esophagogastric junction: a pooled analysis from the international BEACON consortium. $J$ Natl Cancer Inst. 2010;102(17):1344-1353.

7. Toh Y, Oki E, Ohgaki K, et al. Alcohol drinking, cigarette smoking, and the development of squamous cell carcinoma of the esophagus: molecular mechanisms of carcinogenesis. Int J Clin Oncol. 2010;15(2):135-144.

8. Huang FL, Yu SJ. Esophageal cancer: Risk factors, genetic association, and treatment. Asian J Surg. 2018;41(3):210-215.

9. Lam AK. Introduction: Esophageal Squamous Cell Carcinoma-Current Status and Future Advances. Methods Mol Biol. 2020;2129:1-6.

10. Visbal AL, Allen MS, Miller DL, Deschamps C, Trastek VF, Pairolero PC. Ivor Lewis esophagogastrectomy for esophageal cancer. Ann Thorac Surg. 2001;71(6):1803-1808.

11. Collard JM. Exclusive radical surgery for esophageal adenocarcinoma. Cancer. 2001;91(6):10981104.

12. Ning MS, Ahobila V, Jhingran A, et al. Outcomes and patterns of relapse after definitive radiation therapy for oligometastatic cervical cancer. Gynecol Oncol. 2018;148(1):132-138.

13. Hata M, Koike I, Miyagi E, et al. Radiation Therapy for Patients with Bone Metastasis from Uterine Cervical Cancer: Its Role and Optimal Radiation Regimen for Palliative Care. Anticancer Res. 
2018;38(2):1033-1040.

14. Luo YJ, Mao QF, Wang XL, Yu JM, Li MH. Radiotherapy for esophageal carcinoma: dose, response and survival. Cancer Manag Res. 2018;10:13-21.

15. Terry PD, Rohan TE, Wolk A. Intakes of fish and marine fatty acids and the risks of cancers of the breast and prostate and of other hormone-related cancers: a review of the epidemiologic evidence. Am J Clin Nutr. 2003;77(3):532-543.

16. Leitzmann MF, Stampfer MJ, Michaud DS, et al. Dietary intake of n-3 and n-6 fatty acids and the risk of prostate cancer. Am J Clin Nutr. 2004;80(1):204-216.

17. Salehi M, Moradi-Lakeh M, Salehi MH, Nojomi M, Kolahdooz F. Meat, fish, and esophageal cancer risk: a systematic review and dose-response meta-analysis. Nutr Rev. 2013;71(5):257-267.

18. Wang S, Hannafon BN, Wolf RF, et al. Characterization of docosahexaenoic acid (DHA)-induced heme oxygenase-1 (HO-1) expression in human cancer cells: the importance of enhanced BTB and CNC homology 1 (Bach1) degradation. J Nutr Biochem. 2014;25(5):515-525.

19. Jing K, Shin S, Jeong S, et al. Docosahexaenoic acid induces the degradation of HPV E6/E7 oncoproteins by activating the ubiquitin-proteasome system. Cell Death Dis. 2014;5:e1524.

20. Kang KS, Wang P, Yamabe N, Fukui M, Jay T, Zhu BT. Docosahexaenoic acid induces apoptosis in MCF-7 cells in vitro and in vivo via reactive oxygen species formation and caspase 8 activation. PLOS One. 2010;5(4):e10296.

21. Zamani SA, McClain KM, Graubard BI, et al. Dietary polyunsaturated fat intake in relation to head and neck, esophageal, and gastric cancer incidence in the NIH-AARP Diet and Health Study. Am J Epidemiol. 2020.

22. Tontonoz P, Spiegelman BM. Fat and beyond: The diverse biology of PPAR gamma. Annu Rev Biochem. 2008;77:289-312.

23. Mohata S, Kumar HS, Sharma N, Jhakhar SL, Beniwal S, Harsh KK. Acute treatment-related toxicity in elderly patients with good performance status compared to young patients in locally advanced esophageal carcinoma treated by definitive chemoradiation: A retrospective comparative study. J Cancer Res Ther. 2020;16(1):116-119.

24. Backes J, Anzalone D, Hilleman D, Catini J. The clinical relevance of omega-3 fatty acids in the management of hypertriglyceridemia. Lipids Health Dis. 2016;15(1):118.

25. Belkouch M, Hachem M, Elgot A, et al. The pleiotropic effects of omega-3 docosahexaenoic acid on the hallmarks of Alzheimer's disease. J Nutr Biochem. 2016;38:1-11. 
26. Colas S, Maheo K, Denis F, et al. Sensitization by dietary docosahexaenoic acid of rat mammary carcinoma to anthracycline: a role for tumor vascularization. Clin Cancer Res. 2006;12(19):5879-5886.

27. Shin JI, Jeon YJ, Lee S, Lee YG, Kim JB, Lee K. G-Protein-Coupled Receptor 120 Mediates DHAInduced Apoptosis by Regulating IP3R, ROS and, ER Stress Levels in Cisplatin-Resistant Cancer Cells. Mol Cells. 2019;42(3):252-261.

28. Jeong S, Kim DY, Kang SH, et al. Docosahexaenoic Acid Enhances Oxaliplatin-Induced Autophagic Cell Death via the ER Stress/Sesn2 Pathway in Colorectal Cancer. Cancers (Basel). 2019;11(7).

29. Irun P, Lanas A, Piazuelo E. Omega-3 Polyunsaturated Fatty Acids and Their Bioactive Metabolites in Gastrointestinal Malignancies Related to Unresolved Inflammation. A Review. Front Pharmacol. 2019;10:852.

30. Santivasi WL, Xia F. Ionizing radiation-induced DNA damage, response, and repair. Antioxid Redox Signal. 2014;21(2):251-259.

31. Montero JA, Sanchez-Fernandez C, Lorda-Diez Cl, Garcia-Porrero JA, Hurle JM. DNA damage precedes apoptosis during the regression of the interdigital tissue in vertebrate embryos. Sci Rep. 2016;6:35478.

32. Frosina G. DNA repair and resistance of gliomas to chemotherapy and radiotherapy. Mol Cancer Res. 2009;7(7):989-999.

33. Ding WQ, Vaught JL, Yamauchi H, Lind SE. Differential sensitivity of cancer cells to docosahexaenoic acid-induced cytotoxicity: the potential importance of down-regulation of superoxide dismutase 1 expression. Mol Cancer Ther. 2004;3(9):1109-1117.

34. Cai F, Sorg O, Granci V, et al. Interaction of omega-3 polyunsaturated fatty acids with radiation therapy in two different colorectal cancer cell lines. Clin Nutr. 2014;33(1):164-170.

35. Germain E, Chajes V, Cognault S, Lhuillery C, Bougnoux P. Enhancement of doxorubicin cytotoxicity by polyunsaturated fatty acids in the human breast tumor cell line MDA-MB-231: relationship to lipid peroxidation. Int J Cancer. 1998;75(4):578-583.

36. Jiao Y, Hannafon BN, Zhang RR, Fung KM, Ding WQ. Docosahexaenoic acid and disulfiram act in concert to kill cancer cells: a mutual enhancement of their anticancer actions. Oncotarget. 2017;8(11):17908-17920.

37. Kaplan JM, Denenberg A, Monaco M, Nowell M, Wong H, Zingarelli B. Changes in peroxisome proliferator-activated receptor-gamma activity in children with septic shock. Intensive Care Med. 2010;36(1):123-130.

38. Naeini Z, Toupchian O, Vatannejad A, et al. Effects of DHA-enriched fish oil on gene expression levels of p53 and NF-kappaB and PPAR-gamma activity in PBMCs of patients with T2DM: A randomized, 
double-blind, clinical trial. Nutr Metab Cardiovasc Dis. 2020;30(3):441-447.

39. Girnun GD, Smith WM, Drori S, et al. APC-dependent suppression of colon carcinogenesis by PPARgamma. Proc Natl Acad Sci U S A. 2002;99(21):13771-13776.

40. Moldes M, Zuo Y, Morrison RF, et al. Peroxisome-proliferator-activated receptor gamma suppresses Wnt/beta-catenin signalling during adipogenesis. Biochem J. 2003;376(Pt 3):607-613.

41. Smith MR, Manola J, Kaufman DS, et al. Rosiglitazone versus placebo for men with prostate carcinoma and a rising serum prostate-specific antigen level after radical prostatectomy and/or radiation therapy. Cancer. 2004;101(7):1569-1574.

Figures

A

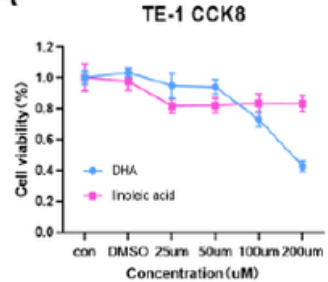

Concentration(uM)

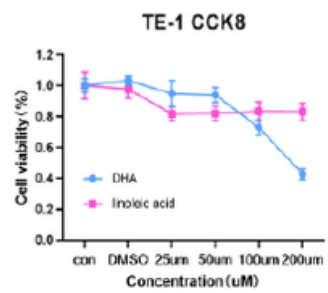

Concentration (UM)

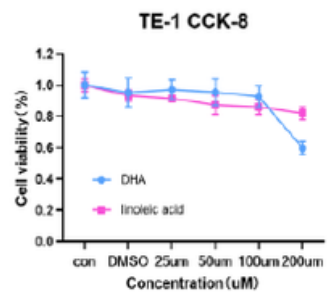

B

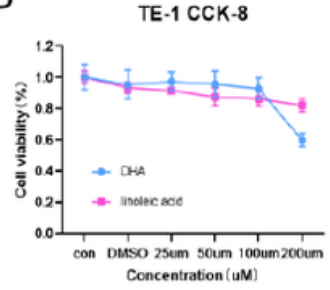

TE-10 CCK-8

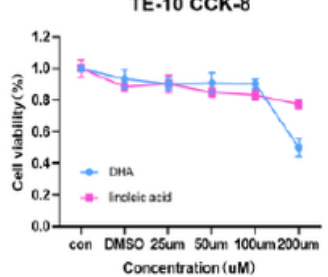

TE-10 CCK-8

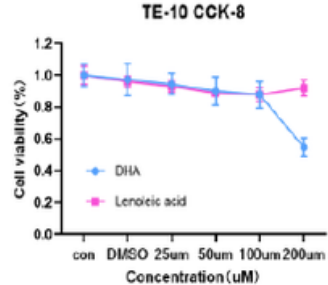

C

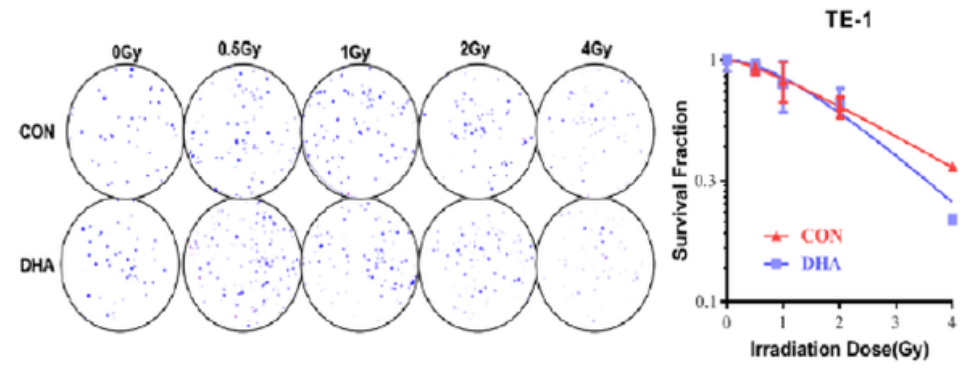

\begin{tabular}{ccccc}
\hline TE-10 & D0 & Dq & N & SER \\
\hline Control & 3.119 & 9.954 & 1.368 & \\
DHA & 2.052 & 3.084 & 1.945 & 1.520 \\
\hline
\end{tabular}

$\mathrm{D}$
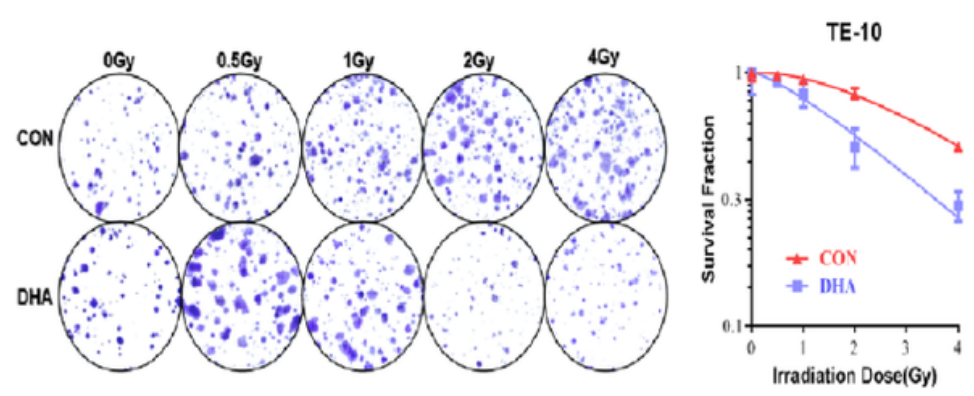

\begin{tabular}{ccccc}
\hline TE-10 & DO & Dq & N & SER \\
\hline Control & 3.014 & 4.036 & 2.339 & \\
DHA & 2.461 & 1.041 & 1.423 & 1.225 \\
\hline
\end{tabular}

\section{Figure 1}

DHA reduces cell viability after irradiation in esophagus cells. TE- 1 and TE-10 cell viability curves coincubation with 0, 25, 50, 100 and 200uM DHA and LNA for 24h. (A) Representative clones from every group after $0,0.5,1,2$ and 4 Gy radiation and the corresponding clonogenic cell survival curves were 
matched for TE-1 and TE-10 esophagus cells that treated with DHA and LNA for 24h before irradiation. (B-C) The survival rates were normalized to the unirradiated cells and the sensitizer enhancement ratio(SER) was measured by the multi-target, single-hit model.
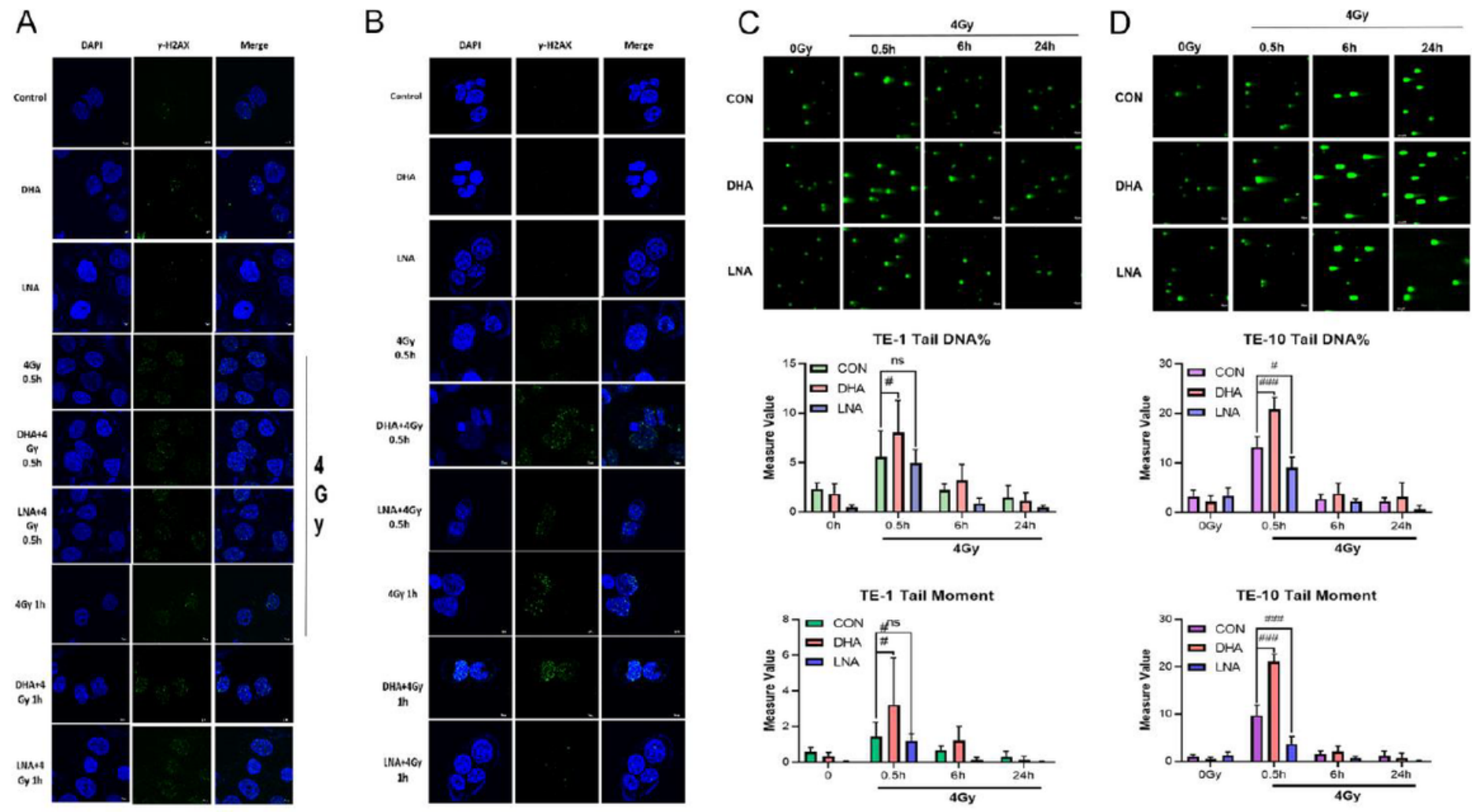

TE-10 Tail DNA\%
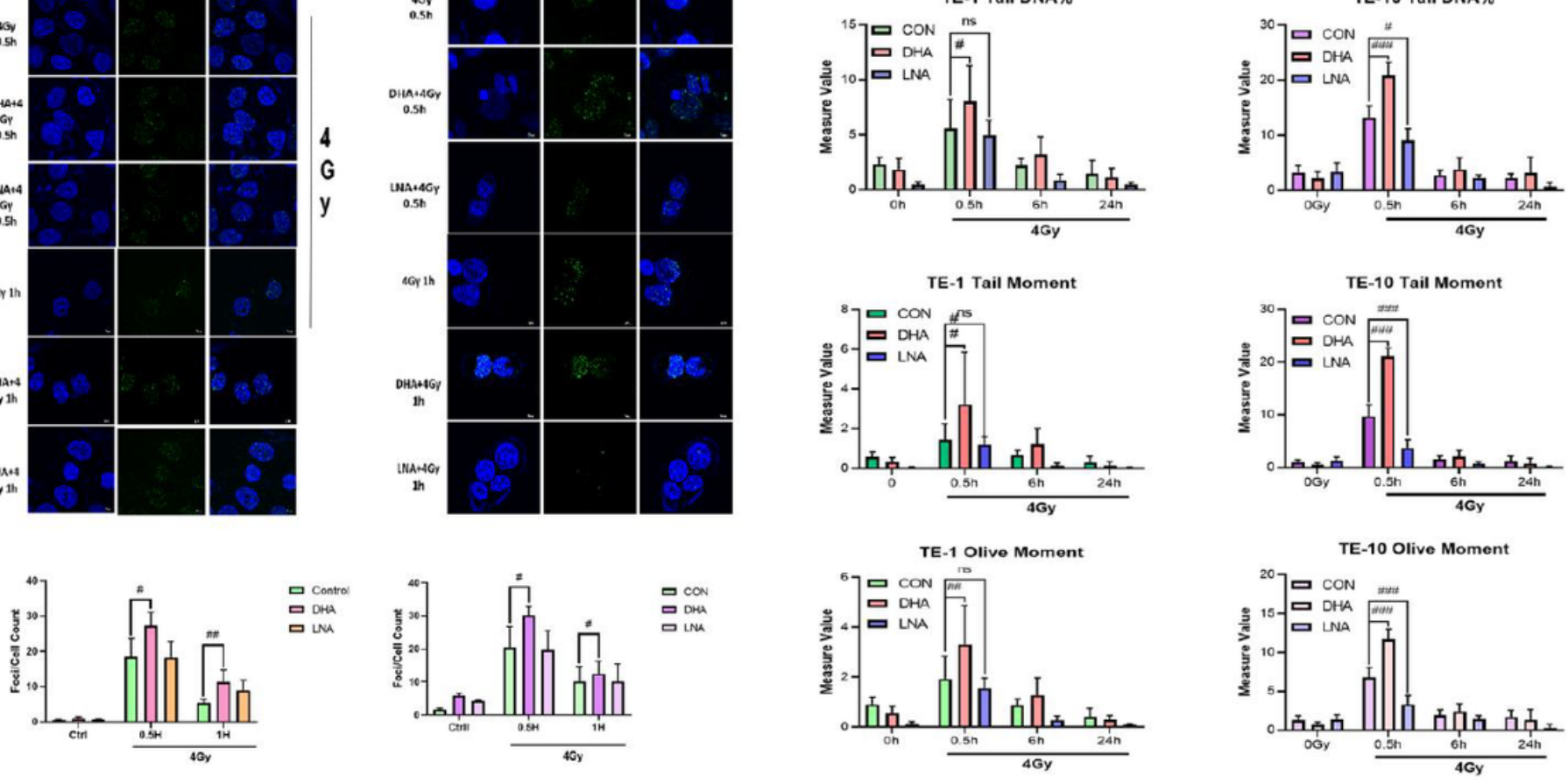

\section{Figure 2}

DHA and IR combination enhances DNA damage in esophagus cancer cells. Immunofluorescences of $Y^{-}$ H2AX staining in TE-1 and TE-10 after co-incubation with DHA and LNA for 24h before get irradiated were taken respectively in $0.5 \mathrm{~h}$ and $1 \mathrm{~h}$. (A-B) the scale bar represents $7 \mu \mathrm{m}$. The comet assay was carried out to measure the DNA damage of IR. Cells were pretreated with DHA and LNA followed by irradiation. Cells were gathered at different point of time after irradiation. (C-D) 

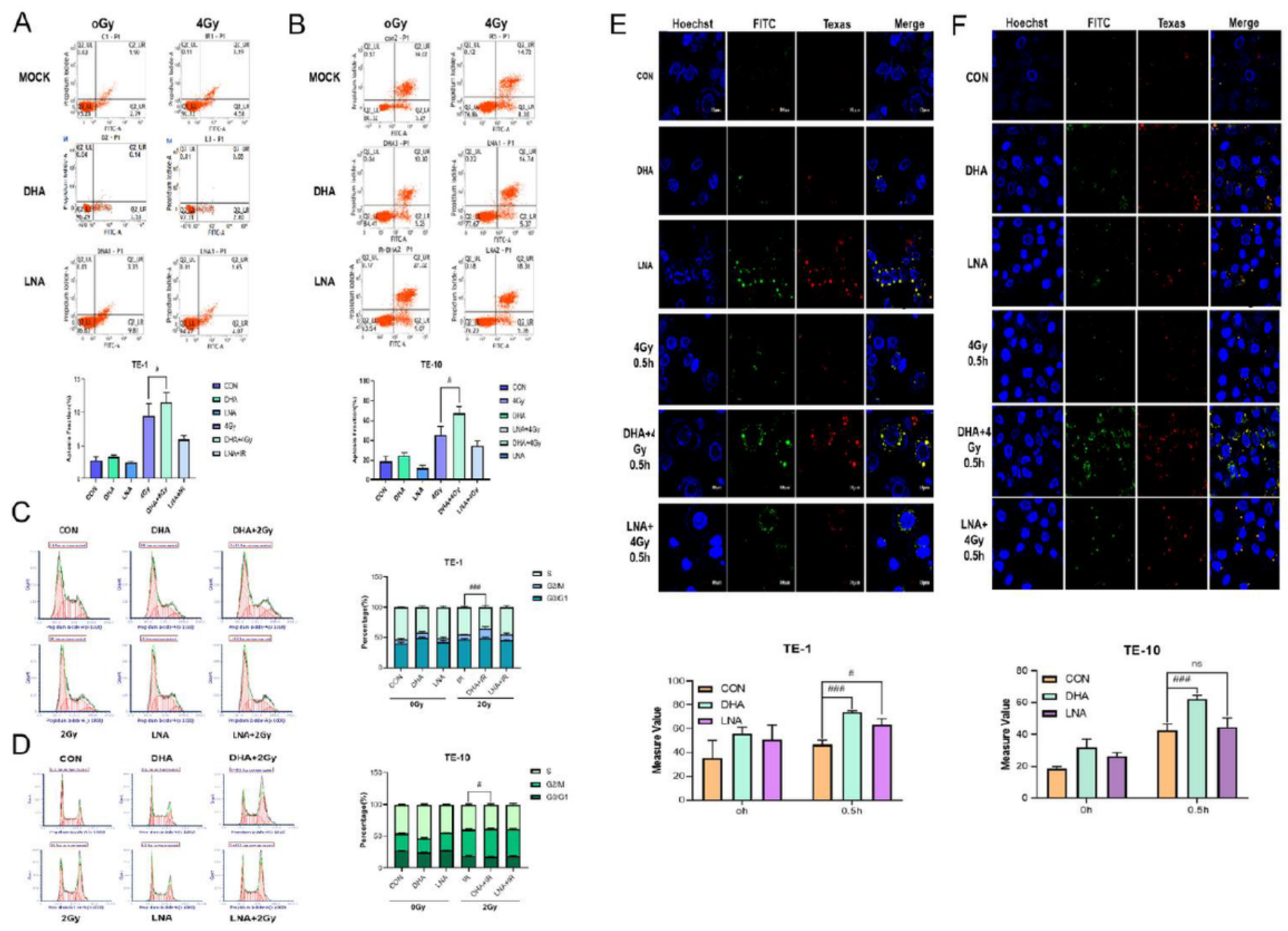

TE-1

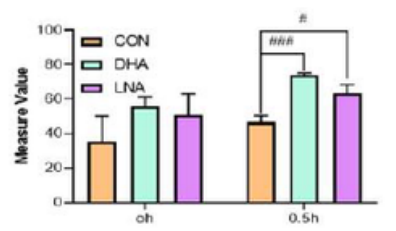

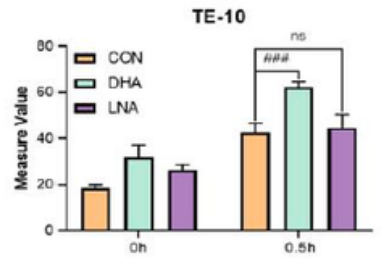

\section{Figure 3}

DHA improved apoptosis caused by IR in esophagus cells. Apoptosis was measured by Annexin V/7-AAD double staining in esophagus cells. The data were exhibited as the mean \pm s.e.m. of three independent experiments. (A-B) Effects of DHA and IR combination on cell cycles were detected by flow cytometry $24 \mathrm{~h}$ after cell getting 2Gy irradiation. Both attached and flowing cells were collected, $\# \mathrm{P}<0.01$ compared with the control group of cells. (C-D) Typical photomicrographs of BODIPY fluorophore 581/591 staining for lipid droplets in cells $0.5 \mathrm{~h}$ postradiotherapy which received 4Gy irradiation right after the culture with DHA and LNA for 24h.(E-F) 
A
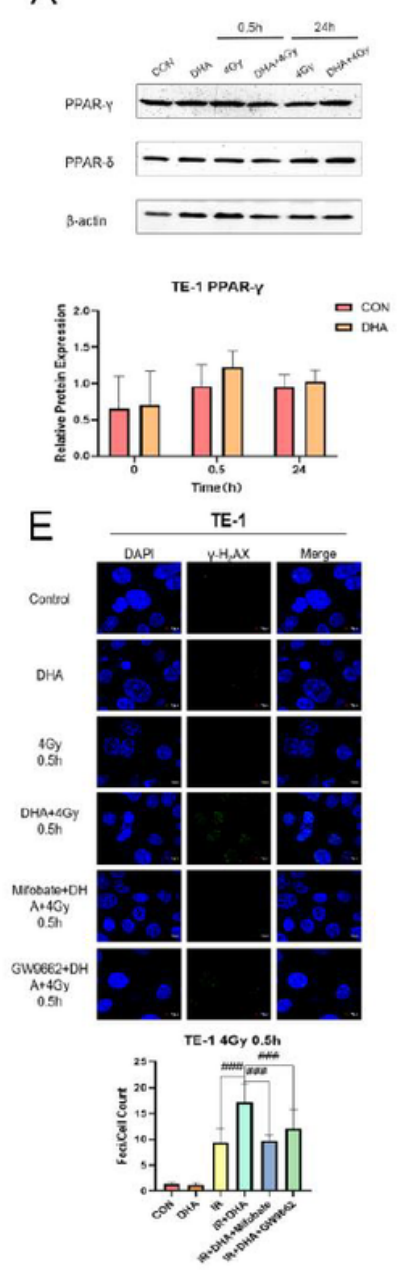

B
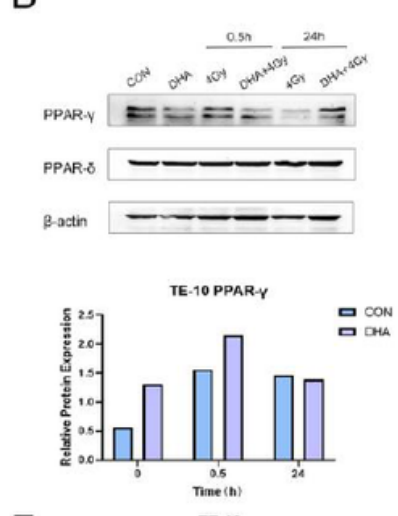

$\mathrm{F}$

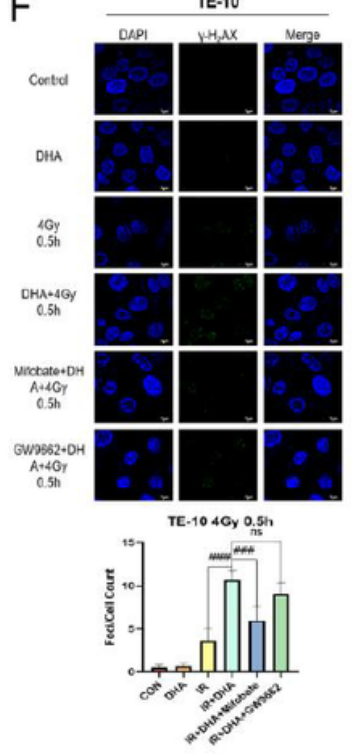

C
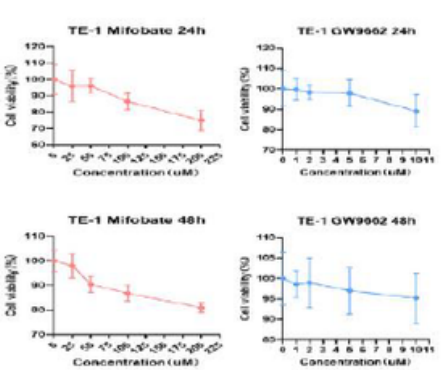

G

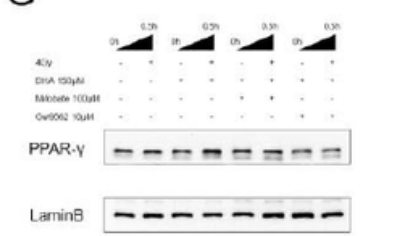

$\mathrm{H}$

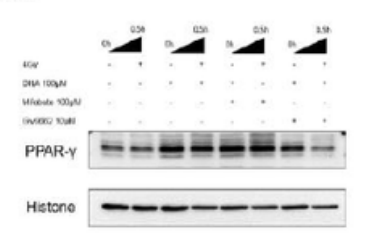

I

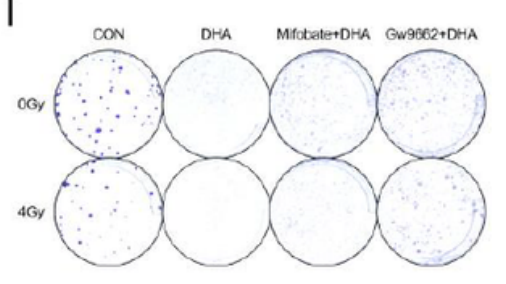

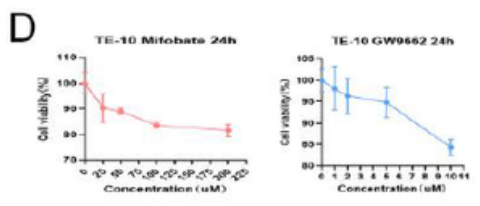
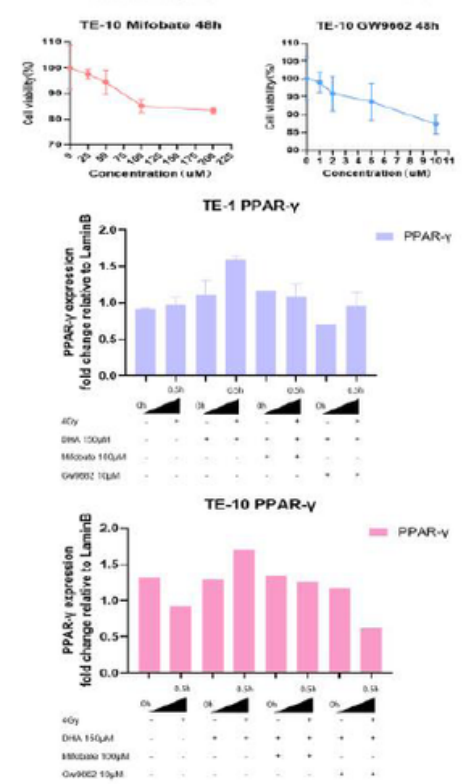

J

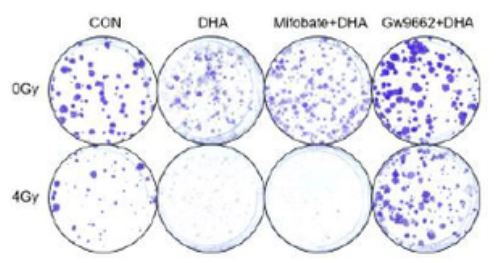

Figure 4

DHA and IR combination augments the expression of PPAR-y. Western blot was performed to determinate the expression of PPAR-Y of TE-1 and TE-10 cells at different point of time after irradiation followed by the quantitative analysis.(A-B) The esophagus cancer were co-incubated with Mifobate and GW9662, cells proliferation inhibition rates were calculated by the CCK-8 assay at $24 \mathrm{~h}$ and $48 \mathrm{~h}$ later.(C-D) DNA damage was verified by $\mathrm{Y}-\mathrm{H} 2 \mathrm{AX}$ using immunofluorescence $0.5 \mathrm{~h}$ after irradiation in TE- 1 and TE-10 cells which got cocultivation with Mifobate and Gw9662 anterior to DHA and IR combination.(E-F) To verify the suppression of PPAR-y induced by the inhibitors Mifobate and Gw9662, westen blot was used to show the nuclear PPAR-y. (G-H) Representative clones from per group with a treatment of 0 or 4 Gy irradiation after the cocultivation of DHA and the PPAR-y inhibitors.(I-J) 
A

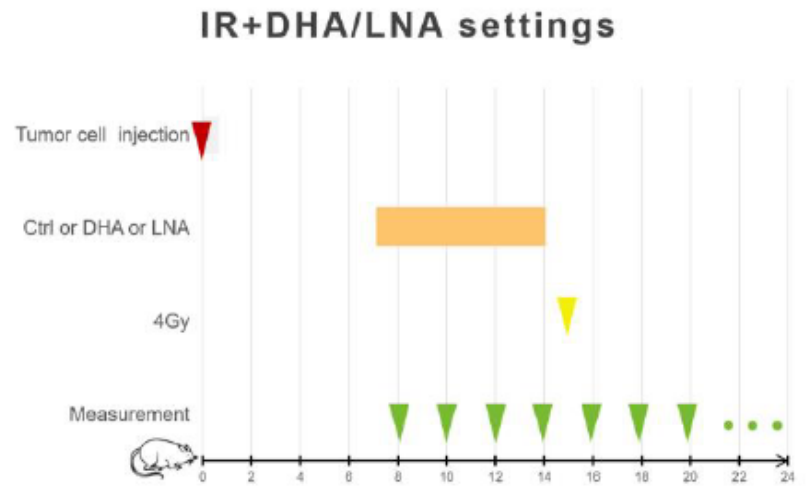

B

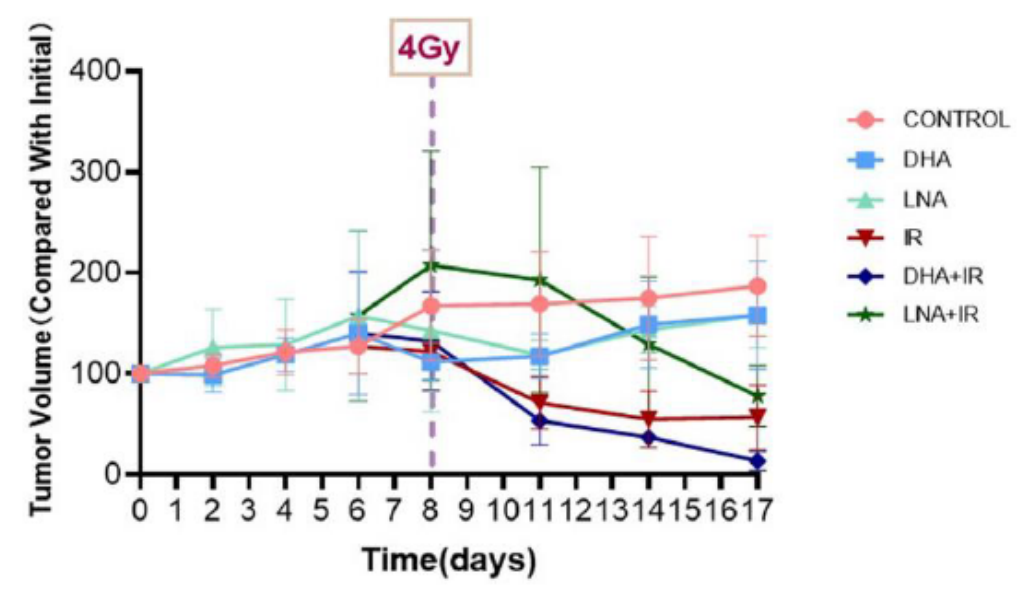

C

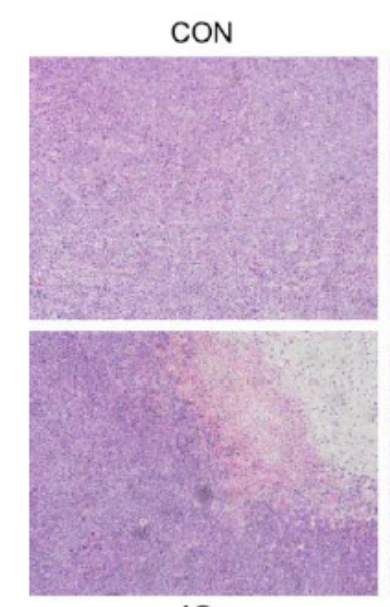

4Gy

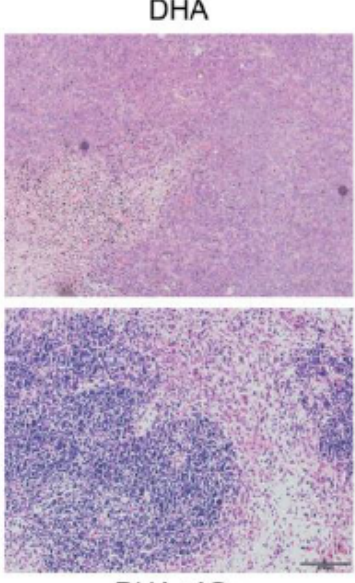

$\mathrm{DHA}+4 \mathrm{~Gy}$
LNA
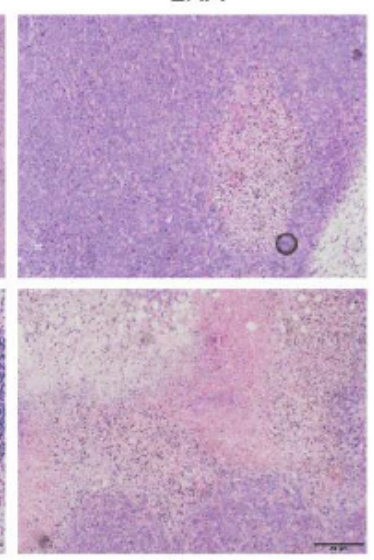

LNA+4Gy

\section{Figure 5}

Every group was consisted of 5 mice. TE-1 cells were inoculated under the skin of the nude mouse. DHA and LNA were given to the mouse by gavage once a day for one week before radiotherapy. (A) The tumor volume was measured daily after TE-1 cells were injected and at 2-day intervals treated with $4 \mathrm{~Gy}$ irradiation. (B) HE staining was used to observe the pathological characteristics of transplantation tumors. (C) 